\title{
Overview on pentaquarks
}

\section{Biplab Dey*}

on behalf of the LHCb collaboration

Central China Normal University, Wuhan, China

E-mail: biplab. dey@cern.ch

An overview of the exotic pentaquark states is provided, from early searches in $K N$ scattering experiments in the 1970's to the now-debunked $\Theta^{+}(1540)$ in the early 2000 's, to the discovery two resonances consistent with pentaquark states by LHCb in 2015 . We review the full angular analysis of $\Lambda_{b}^{0} \rightarrow J / \psi K^{-} p$ that led to affirmation of the resonant nature of the $P_{c}^{+}(4450)$ and $P_{c}^{+}(4380)$ pentaquark candidates. We summarize the latest results, ongoing work and future prospects for other pentaquark searches at $\mathrm{LHCb}$.

The 15th International Conference on Flavor Physics \& CP Violation

5-9 June 2017

Prague, Czech Republic

${ }^{*}$ Speaker. 


\section{Introduction}

The idea that non- $q \bar{q}$ or non- $q q q$ stable, color singlet states are expected to occur have been around since the birth of the quark model in the 1960's [1]. Yet, none of these $q q \bar{q} \bar{q}$ (tetraquark) or $q 9 q q q$ (pentaquark) states were found over the next four decades. The situation changed in 2003 with the Belle discovery of the $X(3872)$ tetraquark candidate, decaying into $J / \psi \pi^{+} \pi^{-}$[2]. Over the next decade, several more tetraquark and pentaquark candidate states have been seen in heavy quark systems (see Ref. [3] for a comprehensive review), but notably none in the light quark sector. The search and study of these states constitute a major thrust at current collider experiments, especially at the LHC, Belle II and BES III.

In these proceedings, after a brief historical overview on pentaquark searches, leading to the first conclusive discovery of pentaquark candidate resonances at LHCb in the $\Lambda_{\mathrm{b}}^{0} \rightarrow J / \psi K^{-} p$ system, we describe some topical details of the amplitude analysis that played a critical role in this discovery. Finally we describe other recent searches at $\mathrm{LHCb}$ and further prospects.

\section{Historical background}

Early searches of pentaquarks in the 1970's focused on the strangeness $S=+1$ baryons, the so-called $Z^{+}$resonances in kaon-nucleon systems (in retrospect, this was before charm was even discovered). For example, "intriguing fluctuations" were seen in LBL [4] $\mathrm{K}^{+}$scattering data on deuteron. Figure 1 shows a re-analysis of the same LBL data in 2003 [5] claiming existence of the $\Theta^{+}(1540)$ pentaquark. By the 1990's, however, interest in these pentaquark claims had waned due to the general skepticism of the partial wave analysis results not being conclusive enough.

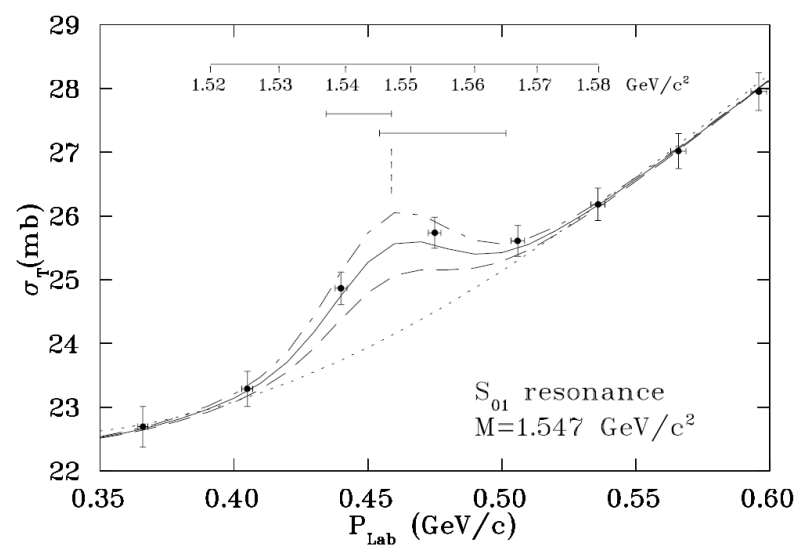

Figure 1: Re-analysis of LBL $K^{+}$-n data [4] by Haidenbauer et al. [5] showing the claimed $\Theta^{+}(1540)$ pentaquark.

The second wave of interest came in 2002 from the LEPS [6] claim of the $\Theta^{+}(1540) \rightarrow K^{+} n$ pentaquark in inclusive $\gamma(n) \rightarrow K^{+} K^{-}(n X)$. The main motivation for this search was a chiralsoliton model based prediction by Polyakov et al. [7] of a $J^{P}=\frac{1}{2}^{+}$narrow $(\Gamma \sim 15 \mathrm{MeV}) u u d d \bar{s}$ $\Theta^{+}$state with a mass around $1530 \mathrm{MeV}$. Between 2002 and 2004, a flurry of both positive and negative results from virtually every running HEP experiment followed (see Ref [8] for a review). 
Finally, in 2006, the CLAS Collaboration published [9] a strong negative result from a dedicated high statistics photoproduction run, that drew curtain on the $\Theta^{+}$saga.

It is worth mentioning here that certain bound meson-baryon molecular states can also be considered as pseudo-pentaquark states. For example, the $\Lambda(1405)$, occurring just below the $N \bar{K}$ threshold has minimal quark content $u s d$, but can also be considered as $u u d s \bar{u}$ in the chiral-unitary model [10] as a dynamically generated $N \bar{K}$ state with two poles. Another example is the $\phi p$ bound state with quark content $s \bar{s} u$, the equivalent of the LHCb hidden-charm $P_{c}^{+}$states in the strange sector [11]. It is interesting to note that both the LEPS [12] and CLAS Collaborations [13] reported a strong local enhancement at $\sqrt{s} \sim 2.1 \mathrm{GeV}$ in photoproduction experiments (see Fig. 2). Since ordinary excited nucleons $\left(N^{*}\right.$ 's) are not anticipated to contain sufficient hidden strangeness, and the dominant production mechanism is by Pomeron exchange, this structure is not expected. Lebed et al. [11] has proposed that a rapidly separating $[s u][\bar{s} u d]$ diquark-antitriquark pair could be the explanation, along the same lines as for the LHCb hidden charm pentaquarks.

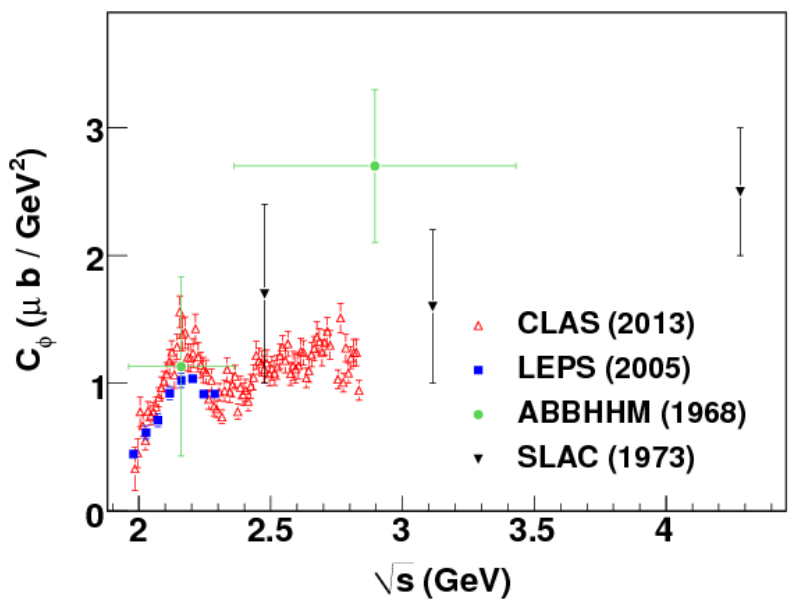

Figure 2: Local enhancement at $\sqrt{s} \sim 2.1 \mathrm{GeV}$ in $\gamma p \rightarrow P_{s}^{+} \rightarrow \phi p$ [13] from a potential $\phi p$ bound state that can be considered as a strange sibling of the $P_{c}^{+}$states [11].

We note here that a recent Belle analysis [14] has examined the $\phi p$ invariant mass in $\Lambda_{c}^{+} \rightarrow$ $\phi p \pi^{0}$. Within statistical limitations, no significant excess is seen in the $m(\phi p) \sim 2 \mathrm{GeV}$ region, but more data from Belle II is awaited.

\section{The LHCb pentaquarks in $\Lambda_{\mathrm{b}}^{0} \rightarrow J / \psi K^{-} p$ and $\Lambda_{\mathrm{b}}^{0} \rightarrow J / \psi \pi^{-} p$}

Among dedicated heavy-flavor physics experiments, the LHCb detector [16] is unique in having access to a wide range of decay modes of numerous $b$-hadron species. $\Lambda_{\mathrm{b}}^{0}$ hadrons are produced copiously in $p p$ interactions at the LHC, and within the LHCb acceptance, the number of $b$-hadron species detected is approximately in the ratio $B: B_{s}^{0}: \Lambda_{\mathrm{b}}^{0}$ is approximately $4: 2: 1$ [17]. Compared to the spin- $0 B$ mesons, $\Lambda_{\mathrm{b}}^{0}$ is particularly interesting due to its spin- $1 / 2$ nature that allows access to spin observables in $b$-decays. The first look at $\Lambda_{\mathrm{b}}^{0} \rightarrow J / \psi K^{-} p$ at LHCb with $1 / \mathrm{fb}$ (2011) data focused on a precise measurement of the $\Lambda_{\mathrm{b}}^{0}$ lifetime [18] where a long-standing discrepancy between the heavy quark effective theory (HQET) expectation of comparable lifetimes among all $b$-hadron species [19] and measurements [20]. While the HQET expectation was confirmed, some structures 
in the $m(J / \psi p)$ variable were visible (see Fig. 3b). Initially, the conjecture was that these artifacts were resulting from $\Lambda^{*} \rightarrow p K^{-}$reflections. However, with the full 3/fb Run I dataset [21], with a very clean sample of $\sim 27000 \Lambda_{\mathrm{b}}^{0}$ decays, a band in $m(J / \psi p)$ is clearly visible in the Dalitz plane, as shown in Fig. 3a.

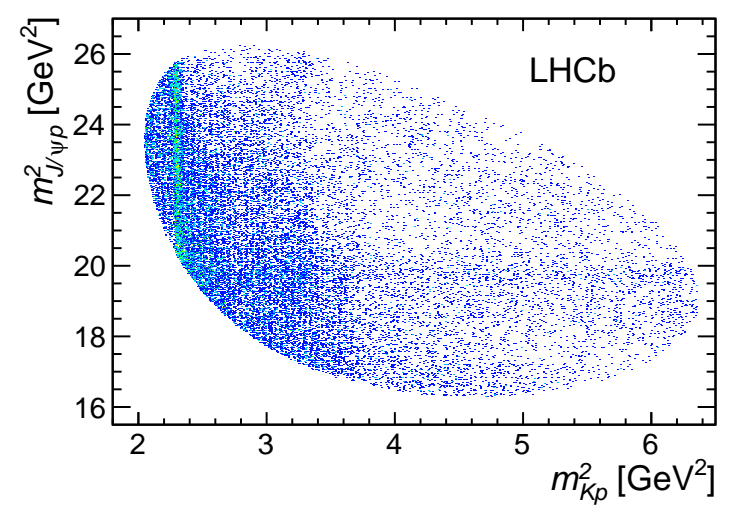

(a)

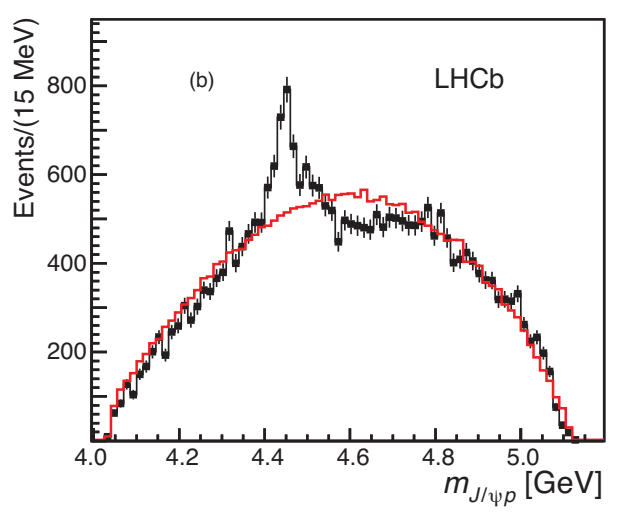

(b)

Figure 3: $\Lambda_{\mathrm{b}}^{0} \rightarrow J / \psi K^{-} p$ : (a) Dalitz distribution in $m^{2}(J / \psi p)$ and $m^{2}\left(p K^{-}\right)$from Ref. [21]. (b) $m(J / \psi p)$ distribution in data (black) compared to expectation from phase-space (red).

Following previous LHCb work on the exotic $Z^{+}$(4430) [22] tetraquark, it was quickly realized that a full angular analysis would be a must to place any pentaquark claim on firm footing. Figure 4 shows the relevant angles:

- $\Lambda_{\mathrm{b}}^{0} \rightarrow J / \psi\left(\rightarrow \mu^{+} \mu^{-}\right) \Lambda^{*}\left(\rightarrow p K^{-}\right)$decay chain: 5 angles $\left\{\theta_{\Lambda_{\mathrm{b}}^{0}}, \theta_{K}, \phi_{K}, \theta_{\psi}, \phi_{\psi}\right\}$ and $m(p K)$, as shown in Fig. 4 a

- $\Lambda_{\mathrm{b}}^{0} \rightarrow P_{c}^{+}\left(\rightarrow J / \psi\left(\rightarrow \mu^{+} \mu^{-}\right) p\right) K^{-}$decay chain: 6 angles $\left\{\theta_{\Lambda_{\mathrm{b}}^{0}}^{P_{c}}, \phi_{P_{c}}, \phi_{\psi}^{P_{c}}, \theta_{P_{c}}, \phi_{\mu}^{P_{c}}, \theta_{\psi}^{P_{c}}, \phi_{\psi}\right\}$ as shown in Fig. $4 \mathrm{~b}$.

The angles $\theta_{\Lambda_{\mathrm{b}}^{0}}^{\left(P_{c}\right)}$ in Fig. 4 arise only when the initial $\Lambda_{\mathrm{b}}^{0}$ is produced polarized, although the production polarization of $\Lambda_{\mathrm{b}}^{0}$ decays in $p p$ collisions is known to be quite small [22].

The analysis employed the helicity formalism to build up sequential decays, where the spinquantization axis for a given decay leg is the flight direction of the mother particle. An additional complication here is that for final-state particles with spin, $\left\{\mu^{ \pm}, p\right\}$, the helicity frames are different between the $\Lambda^{*}$ and $P_{c}^{+}$amplitudes that must be added coherently. Therefore, the spin-quantization axes do not align and an additional rotation is required to align the $\left|\lambda_{\left\{\mu^{ \pm} p\right\}}^{P_{c}}\right\rangle$ helicity basis states in to the $\left|\lambda_{\left\{\mu^{ \pm} p\right\}}^{\Lambda^{*}}\right\rangle$ basis states. For the proton, the rotation angle $\theta_{p}$ is the polar angle between the boost directions of the $\Lambda^{*}$ and $P_{c}^{+}$rest-frames, calculated in the proton rest-frame. For the muons, the helicity direction is the same in both $\Lambda^{*}$ and $P_{c}^{+}$chains, with respect to the mother $J / \psi$ and only the azimuthal angle changes by an amount $\alpha_{\mu}$. The total matrix element reads

$$
|\mathscr{M}|^{2}=\sum_{\lambda_{p}= \pm \frac{1}{2}} \sum_{\Delta \lambda_{\mu}= \pm 1}\left|\mathscr{M}_{\lambda_{p}, \Delta \lambda_{\mu}}^{\Lambda^{*}}+e^{i \Delta \lambda_{\mu} \alpha_{\mu}} \sum_{\lambda_{p}^{P_{c}}= \pm \frac{1}{2}} d_{\lambda_{p}^{P_{c}}, \lambda_{p}}^{\frac{1}{2}}\left(\theta_{p}\right) \mathscr{M}_{\lambda_{p}^{P_{c}}, \Delta \lambda_{\mu}}^{P_{c}}\right|^{2}
$$




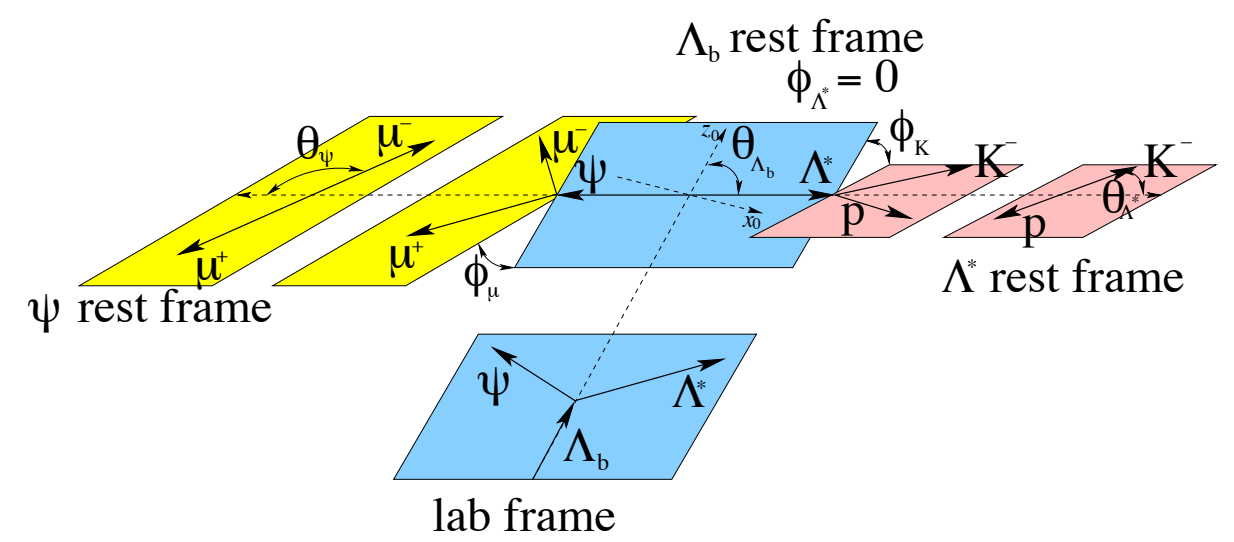

(a)

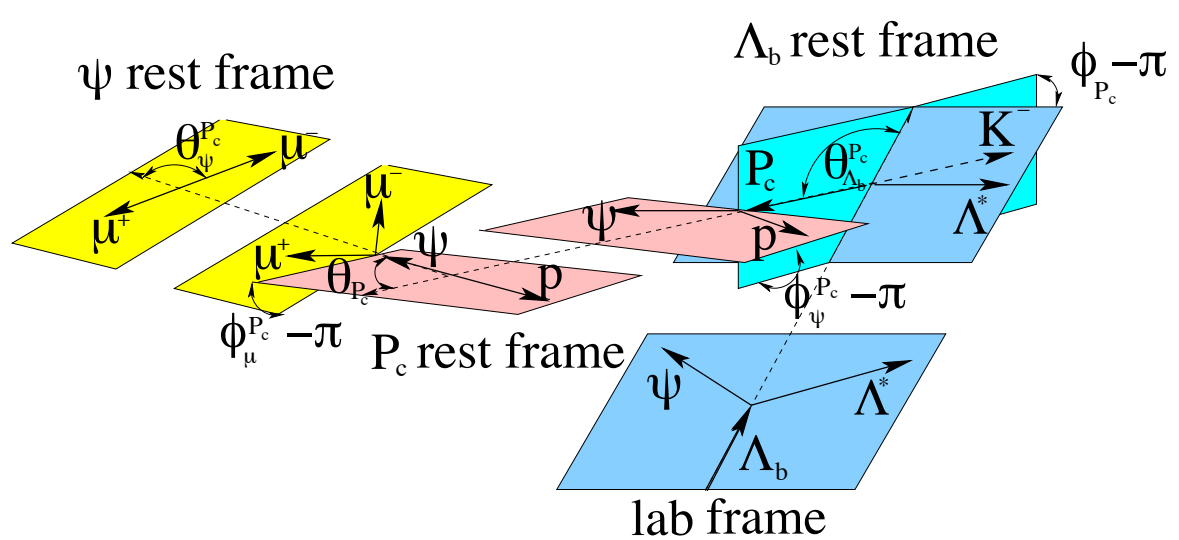

(b)

Figure 4: Angular variables for the (a) $\Lambda_{\mathrm{b}}^{0} \rightarrow J / \psi\left(\rightarrow \mu^{+} \mu^{-}\right) \Lambda^{*}\left(\rightarrow p K^{-}\right)$and (b) $\Lambda_{\mathrm{b}}^{0} \rightarrow P_{c}^{+}(\rightarrow J / \psi(\rightarrow$ $\left.\left.\mu^{+} \mu^{-}\right) p\right) K^{-}$decay chains.

where the $\lambda$ 's denote the various helicities. For the couplings in the $\Lambda^{*}$ and $P_{c}^{+}$decay chains, instead of helicity couplings, the so-called $L S$ couplings were employed. For the two-body decay $A \rightarrow B C$, the outgoing spin is $\vec{S}_{A}=\vec{J}_{B}+\vec{J}_{C}$ with $\left|J_{C}-J_{B}\right| \leq S_{A} \leq\left|J_{C}+J_{B}\right|$, while the total angular momentum is $\vec{J}_{A}=\vec{S}_{A}+\vec{L}_{A}$ and $L_{A}$ is the break-up orbital angular momentum. The helicity basis $\left|\lambda_{B},-\lambda_{C}\right\rangle$ and the $L S$ basis $|L, S\rangle$ are related by Clebsch-Gordon coefficients. The efficacy of the $L S$ formalism is that higher $L_{A}$ partial waves are suppressed and can be ignored in the fits.

Figure 5 shows the projections of the fit results in two variables with only known $\Lambda^{*}$ resonances included. While the $m(p K)$ spectrum is well described, the model clearly disagrees with the data in $m(J / \psi p)$. Figure 6 shows the same with the $P_{c}^{+}$contributions included. The best fit results show strong evidence for a broad $P_{c}^{+}(4380)$ and a narrow $P_{c}^{+}$(4450) consistent with pentaquark candidature, at $9 \sigma$ and $12 \sigma$ significance, respectively. The most-preferred spin-parity configuration is $\left\{\frac{3}{2}^{-}, \frac{5}{2}^{+}\right\}$, while $\left\{\frac{3}{2}^{+}, \frac{5}{2}^{-}\right\}$and $\left\{\frac{5}{2}^{+}, \frac{3}{2}^{-}\right\}$are also possible, the two states always having opposite parities. The phase-motions of the $P_{c}^{+}$amplitudes were also studied: the $P_{c}^{+}(4450)$ phase clearly show a counter-clockwise motion as expected for a true resonance, while, for the $P_{c}^{+}(4380)$, the evidence is somewhat less obvious. 


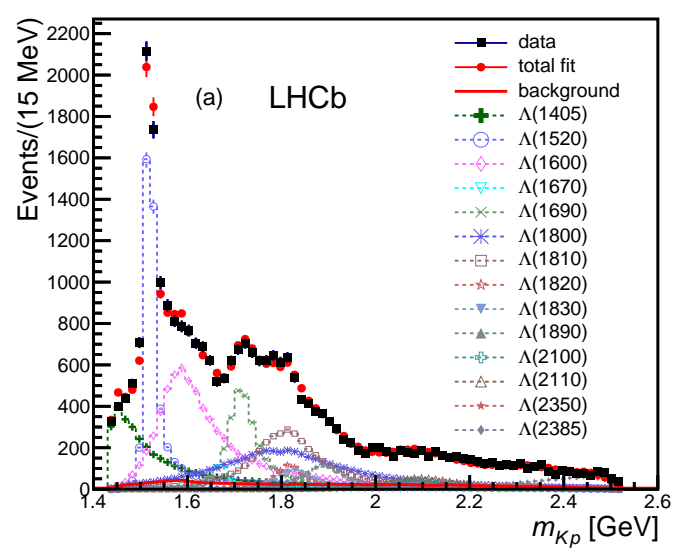

(a)

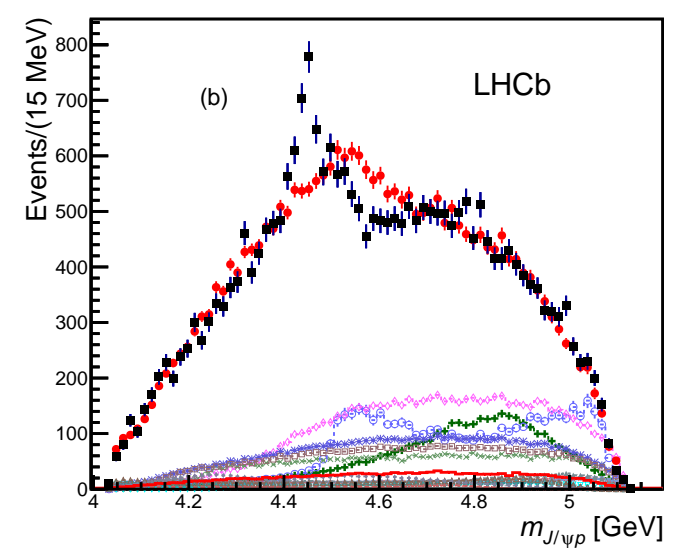

(b)

Figure 5: $\Lambda_{\mathrm{b}}^{0} \rightarrow J / \psi K^{-} p$ fit results with only $\Lambda^{*}$ contributions: (a) $m\left(p K^{-}\right)$and (b) $m(J / \psi p)$ projections.

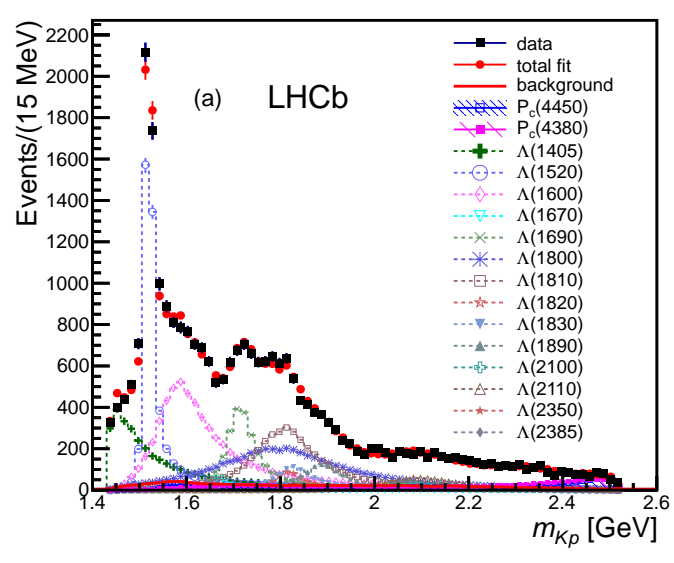

(a)

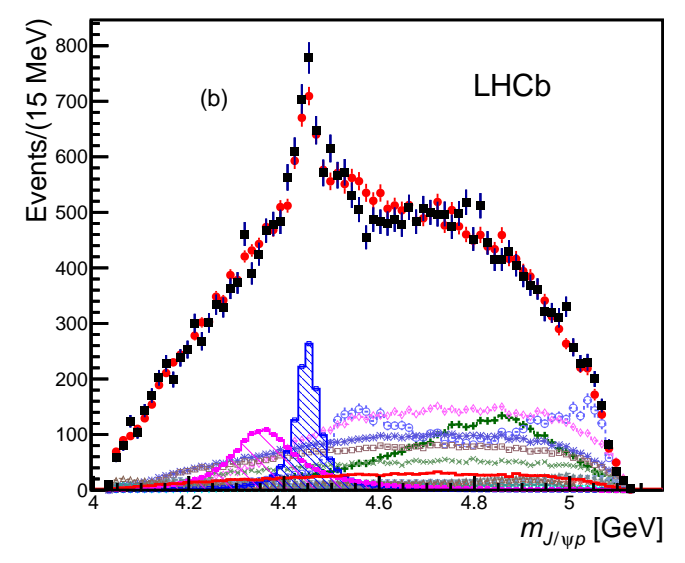

(b)

Figure 6: $\Lambda_{\mathrm{b}}^{0} \rightarrow J / \psi K^{-} p$ fit results with two $P_{c}^{+}$contributions included: (a) $m\left(p K^{-}\right)$and (b) $m(J / \psi p)$ projections. The significances for the $P_{c}^{+}(4380)$ and $P_{c}^{+}(4450)$ are $9 \sigma$ and $12 \sigma$, respectively.

The initial LHCb pentaquark analysis [21] was followed by two more confirmations. First, the Cabibbo suppressed $\Lambda_{\mathrm{b}}^{0} \rightarrow J / \psi \pi^{-} p$ mode [24] with a Run I signal yield of $\sim 1885$ show a $3.1 \sigma$ evidence for presence of exotic contributions, including the $P_{c}^{+}(4380)$ and $P_{c}^{+}(4450)$. In particular, the broad $P_{c}^{+}(4380)$ is more evident here, as shown in Fig. 7a.

Second, the largest systematic uncertainty in the original pentaquark paper was the poorly understood spectrum of the conventional $\Lambda^{*}$ resonances. To overcome this, LHCb studied the hypothesis that the structures in the $m^{2}\left(p K^{-}\right)-m^{2}(J / \psi p)$ Dalitz plane can be explained by $\Lambda^{*} \rightarrow p K^{-}$ decays only [25]. The method relies on constructing a data-driven model out of Legendre polynomial angular moments of the $\Lambda^{*} \rightarrow p K^{-}$decay helicity angle. No detailed knowledge of the underlying $\Lambda^{*}$ spectra is required except that the highest order of the moments be truncated at a reasonable point. On the other hand, reflections from the $P_{c}^{+}$states would lead to moments of un- 
physically high orders. Figure $7 \mathrm{~b}$ shows the projection in $m(J / \psi p)$ from this $\Lambda^{*}$-only model. The overall deviation from the data stands at more than $9 \sigma$, showing in a model-independent fashion that $\Lambda^{*}$ resonances can not explain all features of the data.

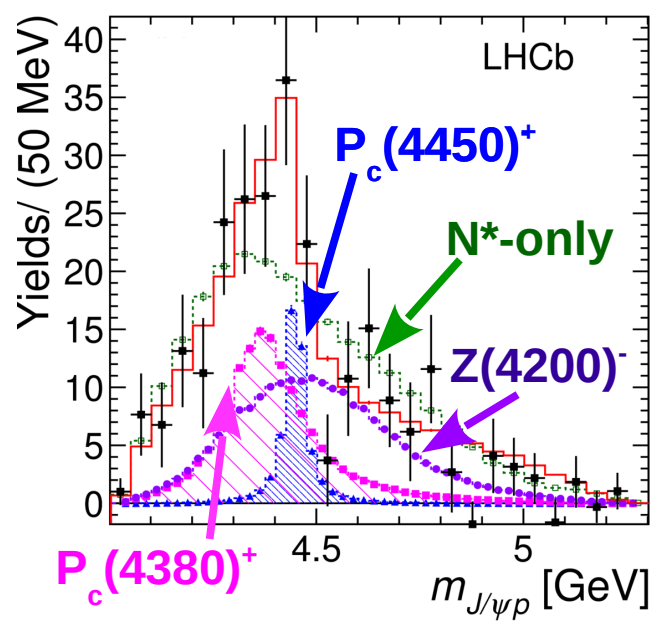

(a)

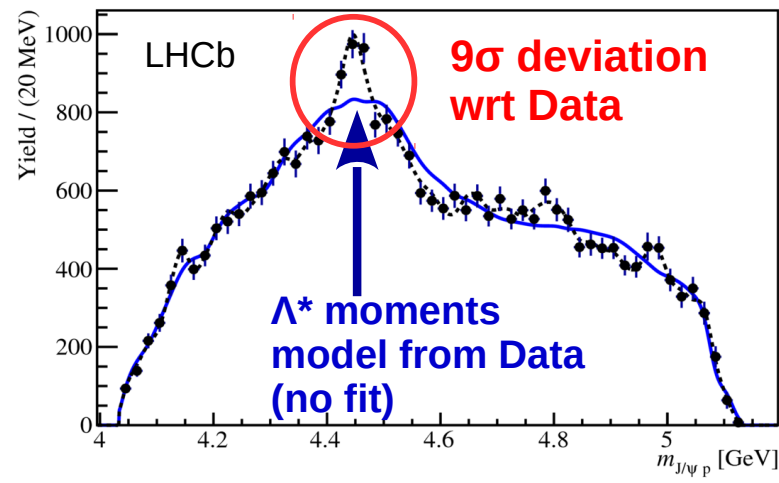

(b)

Figure 7: Further evidence of pentaquarks from LHCb: (a) $P_{c}^{+}$contributions are consistent with $\Lambda_{\mathrm{b}}^{0} \rightarrow$ $J / \psi \pi^{-} p$ data as well [24] (figure covers the $m\left(p \pi^{-}\right)>1.8 \mathrm{GeV}$ region). (b) shows the predictions from the $\Lambda^{*}$ angular moments in $\Lambda_{\mathrm{b}}^{0} \rightarrow J / \psi K^{-} p$ model-independent approach, with a $9 \sigma$ deviation from the data.

\section{Other pentaquark searches at LHCb}

Broadly speaking, ongoing searches at $\mathrm{LHCb}$ are on three fronts:

- new decay modes of the observed $P_{c}^{+}$'s: $\Lambda_{\mathrm{b}}^{0} \rightarrow \chi_{c\{1,2\}} p K^{-}, \Lambda_{\mathrm{b}}^{0} \rightarrow \Lambda_{c}^{+} \overline{D^{0}} K^{-}$.

- new production modes of the observed $P_{c}^{+}: \Lambda_{\mathrm{b}}^{0} \rightarrow J / \psi p K^{*-},\left\{\Upsilon, B_{s}\right\} \rightarrow J / \psi p \bar{p}, \Xi_{b}^{-} \rightarrow$ $J / \psi p K^{-} K^{-}$, inclusive $J / \psi p$.

- new pentaquark multiplets: $\Xi_{b}^{-} \rightarrow J / \psi \Lambda K^{-}, B_{(s)}^{0} \rightarrow J / \psi p \bar{p}, \Lambda_{\mathrm{b}}^{0} \rightarrow J / \psi \Lambda \phi, \Lambda_{\mathrm{b}}^{0} \rightarrow \Sigma_{c}^{++} h^{+} \pi^{-} \pi^{-} h^{-}$, $h \in\{\pi, K\}$.

We cover three of the above topics where Run I results are published.

\subsection{First observation of $\Lambda_{\mathrm{b}}^{0} \rightarrow \chi_{c\{1,2\}} p K^{-}$}

The main motivation for this mode is the observation that the $P_{c}^{+}(4450)$ occurs just above the $\left[\chi_{c 1} p\right]$ threshold and could arise from a rescattering effect [26]. Guo et al [26] showed that the observed phase-motion of the $P_{c}^{+}(4450)$ amplitude is also accountable in this rescattering picture. They also proposed that if the $P_{c}^{+}(4450)$ is a true resonance, a tell-tale sign would be its signature in the $\left[\chi_{c 1} p\right]$ mode itself, which rescattering would not explain as a peak above threshold. The LHCb analysis [27] including the full 3/fb Run I dataset is the first observation of this mode. The decays 
$\chi_{c\{1,2\}} \rightarrow J / \psi \gamma$ were employed, with $\Lambda_{\mathrm{b}}^{0} \rightarrow J / \psi p K^{-}$as the normalization mode. Data-driven techniques were used to correct for data/simulation differences in $\Lambda_{\mathrm{b}}^{0}$ production kinematics, particle identification, and $\chi_{c J}$ polarization. The final $\Lambda_{\mathrm{b}}^{0}$ mass fits with $J / \psi$ and $\chi_{c 1}$ mass-constrained in a kinematic fit are shown in Fig. 8a. Note that the $\chi_{c 1}$ mass constraint pushes the $\Lambda_{\mathrm{b}}^{0} \rightarrow \chi_{c 2} p K^{-}$ contribution to lower masses. Figure $8 \mathrm{~b}$ shows the background-subtracted $\chi_{c J}$ mass spectra with the $\chi_{c 1}$ mass-constraint removed. The signal yields are $453 \pm 25$ and $285 \pm 23$ for the $\chi_{c 1}$ and $\chi_{c 2}$, respectively. The small breakup momenta in these decays allow for a precise measurement of the $\Lambda_{\mathrm{b}}^{0}$ mass as

$$
m\left(\Lambda_{\mathrm{b}}^{0}\right)=5619.44 \pm 0.28(\text { stat }) \pm 0.26(\text { sys }) .
$$

The relative branching fractions are measured as

$$
\frac{\mathscr{B}\left(\Lambda_{\mathrm{b}}^{0} \rightarrow \chi_{c 2} p K^{-}\right)}{\mathscr{B}\left(\Lambda_{\mathrm{b}}^{0} \rightarrow \chi_{c 1} p K^{-}\right)}=1.02 \pm 0.11
$$

which is somewhat at odds with a previous LHCb measurement [30]

$$
\frac{\mathscr{B}\left(B^{0} \rightarrow \chi_{c 2} K^{*}\right)}{\mathscr{B}\left(B^{0} \rightarrow \chi_{c 1} K^{*}\right)}=0.17 \pm 0.05
$$

While the $m\left(\chi_{c J} p\right)$ and $m(p K)$ mass spectra have been investigated, more data will be required. The addition of Run II statistics will facilitate an amplitude analysis, where we note that the radiative photon make the analysis different from $\Lambda_{\mathrm{b}}^{0} \rightarrow J / \psi p K^{-}$.

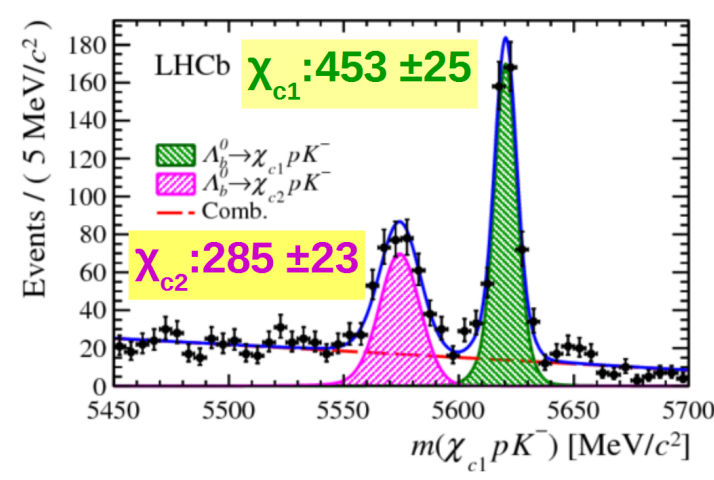

(a)

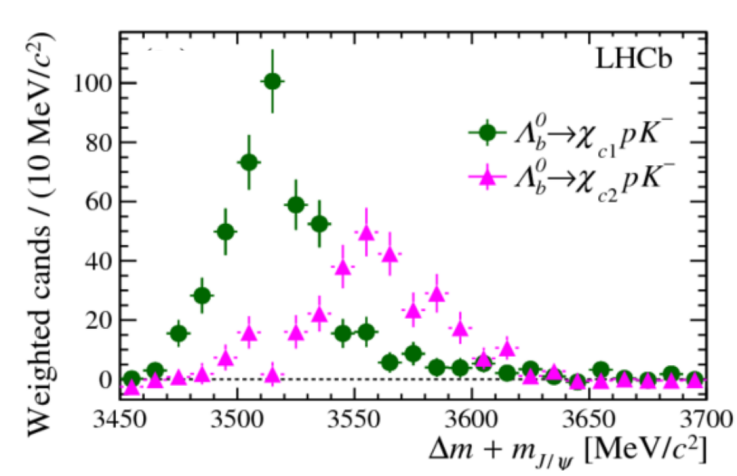

(b)

Figure 8: Mass fits for Run I $\Lambda_{\mathrm{b}}^{0} \rightarrow \chi_{c\{1,2\}} p K^{-}$[27]: (a) with and (b) without the $\chi_{c 1}$ mass-constrained

\subsection{First observation of the decay $\Xi_{b}^{-} \rightarrow J / \psi \Lambda K^{-}$}

In the chiral unitary model of Chen et al. [29], the $P_{c}^{+}(4450)$ is of a molecular nature and implies a strangeness hidden-charm partner of mass $\sim 4650 \mathrm{MeV}$ and width $\sim 10 \mathrm{MeV}$, decaying as $P_{c s} \rightarrow J / \psi \Lambda$. Chen et al. suggested looking at the $\Xi_{b}^{-} \rightarrow J / \psi \Lambda K^{-}$to search for the $P_{c s}$. LHCb has made the first observation of this mode employing the Run I dataset [30]. The long lifetime of the $\Lambda$ results in two separate categories of events depending on whether the $\Lambda$ decays inside (LL) or outside (DD) the innermost vertex detector. The analysis employs $\Lambda_{\mathrm{b}}^{0} \rightarrow J / \psi \Lambda$ as a normalization 


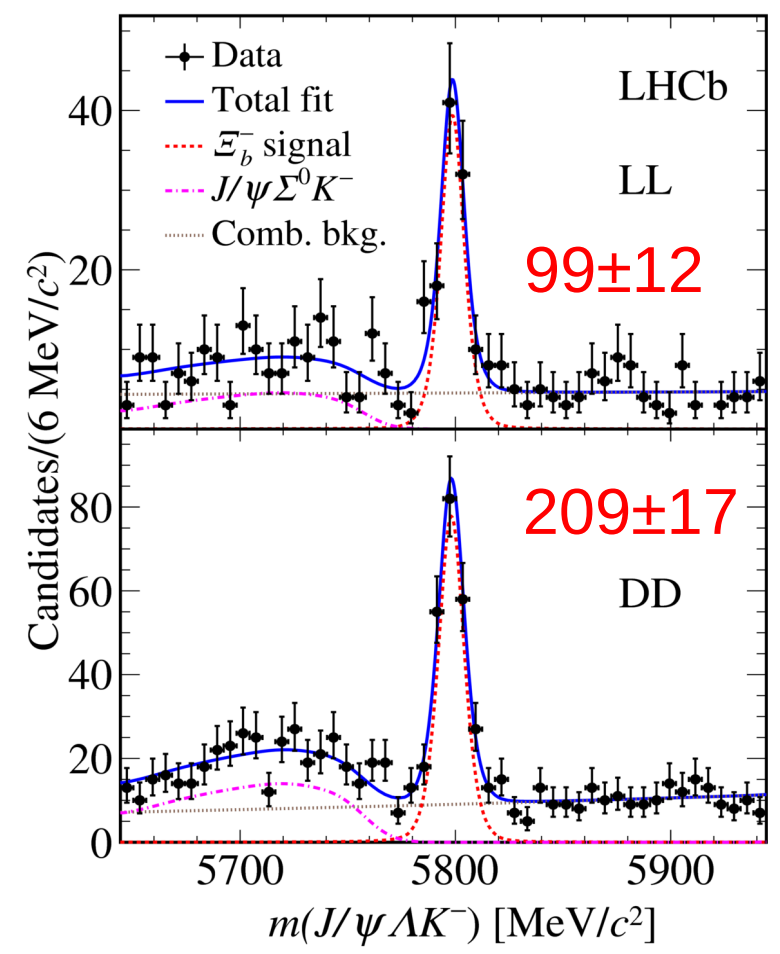

Figure 9: Mass fits for Run I $\Xi_{b}^{-} \rightarrow J / \psi \Lambda K^{-}[30]$ for the LL and DD $\Lambda$ samples.

mode. Figure 9 shows the mass fits and the signal yields. Where $f_{\left\{\Xi_{b}, \Lambda_{\mathrm{b}}^{0}\right\}}$ are the $b \rightarrow\left\{\Xi_{b}, \Lambda_{\mathrm{b}}^{0}\right\}$ are the fragmentation fractions, $\mathrm{LHCb}$ measured:

$$
\frac{f_{\Xi_{b}}}{f_{\Lambda_{\mathrm{b}}^{0}}} \frac{\mathscr{B}\left(\Xi_{b}^{-} \rightarrow J / \psi \Lambda K^{-}\right)}{\mathscr{B}\left(\Lambda_{\mathrm{b}}^{0} \rightarrow J / \psi \Lambda\right)}=0.0419 \pm 0.0029(\text { stat }) \pm 0.0014(\text { sys }) \text {. }
$$

More data will be required to search for the $P_{c s}$.

4.3 Search for $B_{(s)}^{0} \rightarrow J / \psi p \bar{p}$

While phase-space is much more restricted here, with $m(J / \psi p) \leq 4341$ and $4429 \mathrm{MeV}$ for the $B^{0}$ and $B_{s}^{0}$ cases, respectively, at least the $P_{c}^{+}(4380)$ is accessible for the $B_{s}^{0}$ mode. In addition, the ground state pentaquarks can also be searched here. LHCb has analyzed these modes using $1 / \mathrm{fb}$ of Run I data collected in 2011 [31]. The mass fits are shown in Fig. 10. No observation of the decays were seen and the following upper limits were placed

$$
\begin{aligned}
& \mathscr{B}\left(B^{0} \rightarrow J / \psi p \bar{p}\right)<6.0 \times 10^{-7} @ 95 \% \mathrm{CL} \\
& \mathscr{B}\left(B_{s} \rightarrow J / \psi p \bar{p}\right)<5.3 \times 10^{-6} @ 95 \% . \mathrm{CL}
\end{aligned}
$$

The analysis is currently being extended to full Run I and Run II.

\section{Direct photoproduction at JLab}

Direct photoproduction of $\gamma p \rightarrow P_{c}^{+} \rightarrow J / \psi p$ is a natural extension of $\phi p$ photoproduction as described above, from the light $s$-quark to the heavy flavor sector. The $12-\mathrm{GeV}$ upgrade of 


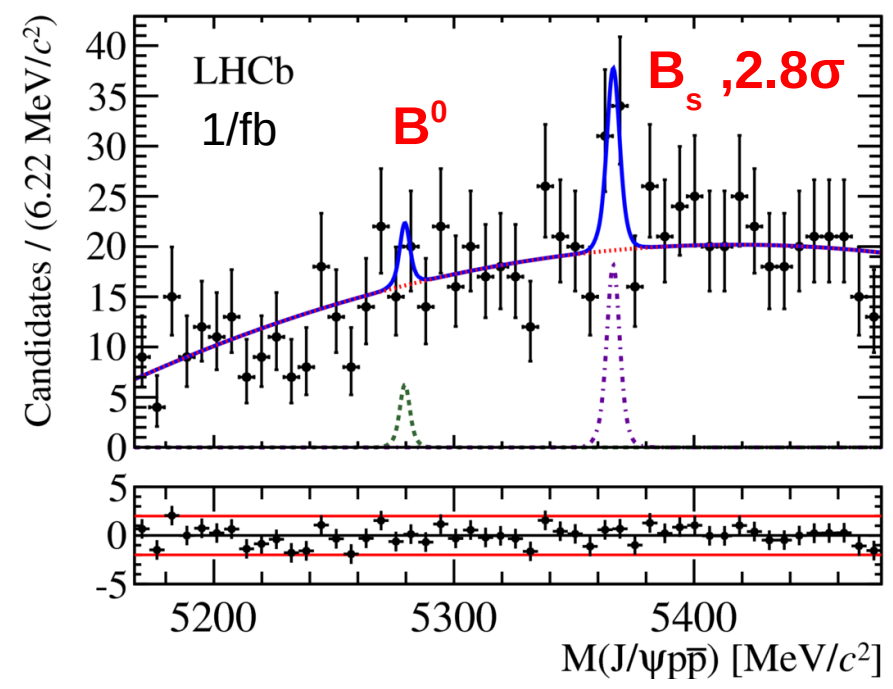

Figure 10: Mass fits for the 1/fb Run I $B_{(s)}^{0} \rightarrow J / \psi p \bar{p}$ LHCb analysis [31].

the CEBAF accelerator is well suited to search for these at JLab using the CLAS12 and GlueX detectors. Several proposals already exist toward this [32, 33, 34]. Figure 11 shows a prediction from Ref. [33], assuming the $P_{c}^{+}$decays only to $J / \psi p$.

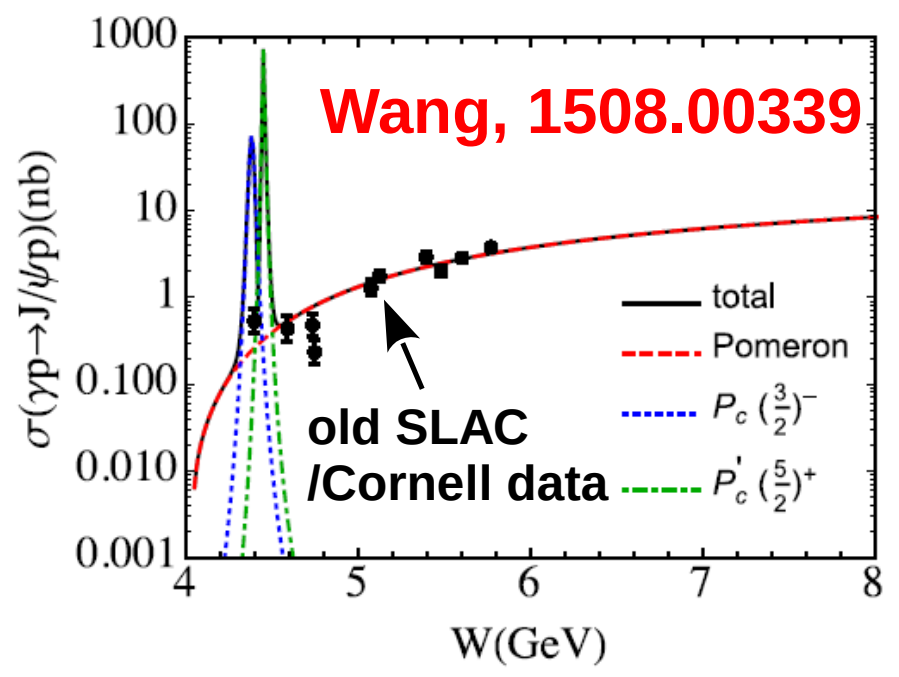

Figure 11: Predictions of the $P_{c}^{+}$photoproduction cross section from Ref. [33].

\section{Summary}

In summary, the $2015 \mathrm{LHCb}$ discovery of two resonant states consistent with pentaquark candidates heralded a new era of our understanding of QCD, especially in the heavy quark sector. It is not clear whether these are genuine five-quark bound states or rescattering effects or molecular 
states. Indeed, according to Karliner and Rosner [35] a whole gamut of double-heavy mesonbaryon molecules could be assessable at the LHC. On the other hand, if these are true pentaquarks, we need to cast a wide net to understand their properties. The spin-parity assignments of the LHCb $P_{c}^{+}$'s are still ambiguous. Complementary observations in other modes and in direct photoproduction, therefore, remain keenly awaited.

\section{References}

[1] Gell-Mann M 1964 Phys. Lett. 8 214-215.

[2] S. K. Choi et al. [Belle Collaboration], Phys. Rev. Lett. 91, 262001 (2003).

[3] R. F. Lebed, R. E. Mitchell and E. S. Swanson, Prog. Part. Nucl. Phys. 93, 143 (2017).

[4] T. Bowen, P. K. Caldwell, F. N. Dikmen, E. W. Jenkins, R. M. Kalbach, D. V. Petersen and A. E. Pifer, Phys. Rev. D 2, 2599 (1970).

[5] J. Haidenbauer and G. Krein, Phys. Rev. C 68, 052201 (2003).

[6] T. Nakano et al. [LEPS Collaboration], Phys. Rev. Lett. 91, 012002 (2003).

[7] D. Diakonov, V. Petrov and M. V. Polyakov, Z. Phys. A 359, 305 (1997).

[8] R. A. Schumacher, AIP Conf. Proc. 842, 409 (2006).

[9] M. Battaglieri et al. [CLAS Collaboration], Phys. Rev. Lett. 96, 042001 (2006).

[10] Y. Kamiya, K. Miyahara, S. Ohnishi, Y. Ikeda, T. Hyodo, E. Oset and W. Weise, Nucl. Phys. A 954, 41 (2016).

[11] R. F. Lebed, Phys. Rev. D 92, no. 11, 114030 (2015).

[12] T. Mibe et al. [LEPS Collaboration], Phys. Rev. Lett. 95, 182001 (2005).

[13] B. Dey et al. [CLAS Collaboration], Phys. Rev. C 89, no. 5, 055208 (2014).

[14] B. Pal et al. [Belle Collaboration], Phys. Rev. D 96, no. 5, 051102 (2017).

[15] J. J. Xie and F. K. Guo, arXiv:1709.01416 [hep-ph].

[16] A. A. Alves, Jr. et al. [LHCb Collaboration], JINST 3, S08005 (2008).

[17] R. Aaij et al. [LHCb Collaboration], Phys. Rev. D 85, 032008 (2012).

[18] R. Aaij et al. [LHCb Collaboration], Phys. Rev. Lett. 111, 102003 (2013).

[19] H. Y. Cheng, Phys. Rev. D 56, 2783 (1997).

[20] E. Franco, V. Lubicz, F. Mescia and C. Tarantino, Nucl. Phys. B 633, 212 (2002).

[21] R. Aaij et al. [LHCb Collaboration], Phys. Rev. Lett. 115, 072001 (2015).

[22] R. Aaij et al. [LHCb Collaboration], Phys. Rev. Lett. 112, no. 22, 222002 (2014).

[23] R. Aaij et al. [LHCb Collaboration], Phys. Lett. B 724, 27 (2013).

[24] R. Aaij et al. [LHCb Collaboration], Phys. Rev. Lett. 117, no. 8, 082003 (2016).

[25] R. Aaij et al. [LHCb Collaboration], Phys. Rev. Lett. 117, no. 8, 082002 (2016).

[26] F. K. Guo, U. G. Meißner, W. Wang and Z. Yang, Phys. Rev. D 92, no. 7, 071502 (2015). 
[27] R. Aaij et al. [LHCb Collaboration], Phys. Rev. Lett. 119, no. 6, 062001 (2017).

[28] R. Aaij et al. [LHCb Collaboration], Nucl. Phys. B 874, 663 (2013).

[29] H. X. Chen, L. S. Geng, W. H. Liang, E. Oset, E. Wang and J. J. Xie, Phys. Rev. C 93, no. 6, 065203 (2016).

[30] R. Aaij et al. [LHCb Collaboration], Phys. Lett. B 772, 265 (2017).

[31] R. Aaij et al. [LHCb Collaboration], JHEP 1309, 006 (2013).

[32] V. Kubarovsky and M. B. Voloshin, Phys. Rev. D 92, no. 3, 031502 (2015).

[33] Q. Wang, X. H. Liu and Q. Zhao, Phys. Rev. D 92, 034022 (2015).

[34] M. Karliner and J. L. Rosner, Phys. Lett. B 752, 329 (2016).

[35] M. Karliner and J. L. Rosner, Phys. Rev. Lett. 115, no. 12, 122001 (2015). 